\title{
COMPARAÇÃO DE MEDIDAS DA CAPACIDADE TAMPONANTE DE MATÉRIAS- PRIMAS E DIETAS UTILIZADAS PARA LEITÕES
}

\section{(Comparison of buffering capacity measures on feedstuffs and diets for piglets)}

\author{
BOCKOR, L. ${ }^{1}$; DAHLKE, F. ${ }^{2}$; MAIORKA, A. ${ }^{2}$; CASTRO, O. S. ${ }^{3}$; OLIVEIRA, E. G. ${ }^{2}$; \\ KRABBE, E. L. ${ }^{4}$; WARPECHOWSKI, M. B. ${ }^{2 *}$
}

${ }^{1}$ Mestranda do Curso de Pós-Graduação em Ciências Veterinárias, Universidade Federa do Paraná, Curitiba, PR. ${ }^{2}$ Departamento de Zootecnia, UFPR, Curitiba, PR. ${ }^{3}$ Acadêmico de Zootecnia, UFPR, Curitiba, PR. ${ }^{4}$ Kemin do Brasil *Autor para Correspondência: Departamento de Zootecnia, Setor de Ciências Agrárias, UFPR. Rua dos Funcionários, 1540, Curitiba, PR. CEP: 80035-050. E-mail: marson@ufpr.br

\begin{abstract}
RESUMO - Avaliou-se a capacidade tamponante (CT) de diferentes ingredientes e dietas para leitões, por meio de diferentes medidas: $\mathrm{pH}$ inicial $(\mathrm{pHi})$, acidez ou alcalinidade tituláveis (AT) e CT. Foram utilizadas 32 amostras de diferentes ingredientes, pré-misturas e dietas, classificadas como aminoácidos, suplementos minerais, cereais e subprodutos, soja e subprodutos, ingredientes de origem animal e dietas completas. $\mathrm{O}$ pHi foi mensurado em três soluções com $10 \mathrm{~g}$ de amostra diluídas em $90 \mathrm{ml}$ de água destilada e deionizada, nas quais foi adicionado ácido clorídrico, ou ácido fórmico ou um acidificante comercial à base de ácido fórmico, para reduzir o $\mathrm{pH}$ até 5,0 , ou $\mathrm{NaOH}$, quando o $\mathrm{pHi}$ foi menor que 5,0 . A AT foi definida como a quantidade de ácido ou base necessária para alterar o $\mathrm{pH}$ entre o $\mathrm{pHi}$ e 5,0, expresso em $\mathrm{mEq} / 100 \mathrm{~g}$ de matéria seca de amostra. A CT foi calculada dividindo-se os valores de AT pelo intervalo de $\mathrm{pH}$ considerado, sendo os valores obtidos com cada ácido considerados replicatas de cada amostra. Os resultados com cada medida foram submetidos à ANOVA considerando as classificações. A correlação entre o pHi e a CT foi também avaliada. Os suplementos minerais e os aminoácidos apresentaram valores extremos em todas as medidas, e foram separados em subgrupos. As classes soja, cereais e dietas mostraram valores similares de $\mathrm{pHi}$, mas diferiram quanto às outras medidas $(P<0,05)$. A CT demonstrou mais sensibilidade que a AT. O pHi apresentou correlação inconsistente com a CT, não sendo uma estimativa confiável desta propriedade físico-química.
\end{abstract}

Palavras-chave: acidez titulável; ácidos orgânicos; alcalinidade titulável; capacidade tamponante; ingredientes.

\begin{abstract}
Buffering capacity (BC) of several feeds and diets for pigs was evaluated by different ways: initial $\mathrm{pH}(\mathrm{pHi})$, titratable acidity or alkalinity (AT) and BC. For the experiment were used 32 samples of different ingredients, mineral supplement mix and diets, classified as amino acids, mineral supplements, cereals and its raw products, soybean and its raw products, animal products, and complete diets. PHi was measured in three solutions with 10 $\mathrm{g}$ sample diluted in $90 \mathrm{ml}$ distilled deionized water, in which one either chloridric acid, formic acid or a commercial acidifier based in formic acid, were used to reduce the $\mathrm{pH}$ just to 5.0 , or $\mathrm{NaOH}$, when the pHi was lower than 5.0. AT was defined as the overall acid or alkali amount required to change the $\mathrm{pH}$ between the $\mathrm{pHi}$ and 5.0, and was expressed as $\mathrm{mEq} / 100 \mathrm{~g}$ sample dry matter. CT was calculated by dividing the AT values by the whole $\mathrm{pH}$ range, with the values obtained with each acid source used as replicates of each sample. Results of each measure were submitted to an ANOVA considering the classifications. Linear correlation between the $\mathrm{pHi}$ and $\mathrm{BC}$ was also evaluated. Minerals and amino acids showed extreme higher and lower values in all measures and were divided in subgroups. Diets and products of soybean and cereal showed similar $\mathrm{pHi}$ values, but were different by the other measures $(P<0,05)$. CT showed higher sensibility than AT. $\mathrm{PHi}$ displayed inconsistent correlation with $\mathrm{BC}$, and was considered a not trustable estimate of that physicochemical property.
\end{abstract}

Key-words: buffering capacity; organic acids; feedstuffs; titratable acidity; titratable alkalinity. 


\section{INTRODUÇÃO}

A fase da desmama é caracterizada pela baixa habilidade dos leitões de secretarem quantidade de $\mathrm{HCl}$ capaz de baixar o $\mathrm{pH}$ gástrico e inibir a proliferação microbiana (Risley et al., 1992). Até esta fase, a acidificação estomacal ocorre primordialmente pela produção de ácido lático a partir da fermentação da lactose do leite ingerido, sendo que altos níveis de lactose no estômago inibem a secreção de $\mathrm{HCl}$ (LAWLOR et al., 2005). De forma inversa, a ingestão de alimento sólido reduz a produção de ácido lático no estômago e estimula a secreção de $\mathrm{HCl}$. Porém, na prática, o consumo de alimento é baixo e variável a desmama. Assim, a combinação de baixa secreção de $\mathrm{HCl}$ e a ingestão variável de alimento, pode resultar em pH elevado, próximo a 5 , podendo permanecer deste modo por vários dias (LAWLOR et al., 2005). Além disso, a capacidade tamponante (CT) dos alimentos pode interferir no $\mathrm{pH}$ estomacal, aumentando a quantidade necessária de $\mathrm{HCl}$ para reduzir o $\mathrm{pH}$ (PENZ, 1991).

Segundo PENZ (1991), a utilização de ácidos orgânicos na dieta de leitões se justifica pela redução do $\mathrm{pH}$ estomacal, aumento na atividade de determinadas enzimas, melhora na digestibilidade de alguns nutrientes e a redução no crescimento de microorganismos. Porém PARTANEN et al. (2002) citam que a composição da dieta pode influenciar a eficácia dos ácidos orgânicos. Da mesma forma ROTH (2000) afirma que em condições de armazenamento inadequadas, o $\mathrm{pH}$ dos alimentos e sua CT diminuem a ação desses ácidos.

Conceitualmente a CT é a habilidade de uma solução resistir à troca de pH (BACCAN et al., 1979). Em nutrição animal, a CT tem sido definida como a habilidade em suspensão aquosa de um alimento em resistir à mudança de $\mathrm{pH}$ decorrente da adição de uma solução ácida ou básica (MCBURNEY et al.,
1983; GABERT et al., 1995; GIGER-REVERDIN et al., 2002).

Diversos métodos têm sido adotados para medir a CT de alimentos para animais (VAN OOIJEN, 2002; MCBURNEY et al., 1983; GABERT et al., 1995; GIGER-REVERDIN et al., 2002; WARPECHOWSKI e CIOCCA, 2006; WARPECHOWSKI e SILVA, 2006; OLIVEIRA Jr. e WARPECHOWSKI, 2007). Porém, ainda não existe consenso sobre o método mais eficiente que possa ser utilizado como padrão para este tipo de mensuração.

VAN OOIJEN (2002) descreve como $b$-value a quantidade $(\mathrm{mEq})$ de $\mathrm{HCl}$ 1,0 M necessária para acidificar um quilograma de alimento até $\mathrm{pH} 4,0$ ou 5,0 . Segundo este autor, o método usualmente utilizado na avaliação de alimentos para suínos é realizado a partir da titulação até o pH 5,0 de 10 gramas de ingrediente suspenso em $90 \mathrm{ml}$ de água destilada. De acordo com BACCAN et al. (1979), a quantidade de ácido ou base necessária para alterar o $\mathrm{pH}$ de uma solução ou suspensão dentro de uma faixa de $\mathrm{pH}$ pré-determinada é chamada de acidez ou alcalinidade tituláveis (AT), e a CT é obtida dividindo-se a AT pelo intervalo total de $\mathrm{pH}$ considerado.

O objetivo deste trabalho foi avaliar diferentes medidas da CT de ingredientes e dietas para leitões.

\section{MATERIAIS E MÉTODOS}

Foram coletadas em fábricas de ração, 32 amostras de ingredientes e dietas comumente utilizados para alimentação de suínos. As amostras foram moídas em moinho tipo WILEY MILL com peneira de $1 \mathrm{~mm}$, e armazenadas em sala com controle de temperatura e umidade, por um período de até três meses. O material experimental foi agrupado em diferentes classes, como apresentado no QUADRO 1.

QUADRO 1 - INGREDIENTES E CLASSIFICAÇÃO UTILIZADA.

\begin{tabular}{ll}
\hline \multicolumn{1}{c}{ Classe } & \multicolumn{1}{c}{ Ingredientes } \\
\hline Soja e subprodutos & Farelo de soja, soja integral e soja integral tostada. \\
Cereais e subprodutos & Milho moído, farelo de arroz integral, farelo de arroz desengordurado e \\
Ingredientes de origem & quirera de arroz. \\
animal & Farinha de penas, farinha de vísceras, farinha de carne, plasma san- \\
Aminoácidos & guíneo e soro de leite em pó. \\
Lisina, metionina e treonina. \\
Suplementos minerais & NaCl, fosfato bicálcico, mistura vitamínica e mineral \\
pré-inicial (Premix1) e crescimento (Premix2). & Dieta pré-inicial peletizada, Dieta crescimento peletizada e \\
& o resíduo de biscoito *
\end{tabular}

${ }^{*} \mathrm{O}$ ingrediente resíduo de biscoito foi incluído na classe dieta por ser um produto resultante da mistura de vários ingredientes. 
Comparação de medidas da capacidade tamponante de

matérias-primas e dietas utilizadas para leitões

A composição das dietas e misturas vitamínicas e minerais analisadas são apresentadas no QUADRO 2. Para a determinação do $\mathrm{pH}$ inicial $(\mathrm{pHi})$ e titulação utilizou-se a metodologia adotada por GABERT et al. (1995), com as seguintes modificações: foram pesadas $10 \mathrm{~g}$ de amostra previamente moída $(<1 \mathrm{~mm})$ para a diluição em $90 \mathrm{~g}$ de água destilada e deionizada, e o $\mathrm{pH}$ foi mensurado com medidor de $\mathrm{pH}(\mathrm{pH}$ 330:SET, WTW), sob agitação magnética constante, em temperatura ambiente. Quando o $\mathrm{pHi}$ foi superior a 5,0, adicionou-se gradativamente a solução

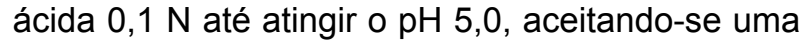
variação de $+/-0,05$ unidades. A mistura foi mantida sob agitação por 15 min e, quando o pH da solução se alterou em mais de 0,1 unidades, a titulação foi continuada, até estabilização do pH. Com soluções de substrato que apresentaram pHi abaixo de 5,0, o mesmo procedimento foi realizado com solução de $\mathrm{NaOH} 0,1 \mathrm{~N}$. Os ácidos utilizados foram o $\mathrm{HCl}$, o ácido fórmico $\left(\mathrm{CH}_{2} \mathrm{O}_{2}\right)$ e um acidificante comercial a base de ácido fórmico (98\% diformiato de potássio, $1,5 \%$ silicato, $0,5 \%$ água - FORMI $^{\circledR}$ ).

\section{QUADRO 2 - COMPOSIÇÃO DAS MISTURAS VITAMÍNICAS E MINERAIS E DAS DIETAS ANALISADAS.}

\begin{tabular}{|c|c|}
\hline Mistura/Dieta & Composição \\
\hline $\begin{array}{l}\text { Mistura vitamínica e mineral } \\
\text { para a fase pré-inicial }\end{array}$ & $\begin{array}{l}\text { Ácido fólico, } 420 \text { mg; ácido pantotênico, } 7,350 \text { mg; antioxidante, } 1.500 \\
\text { mg; biotina, } 16,56 \mathrm{mg} \text {; colina, } 126 \mathrm{~g} \text {; niacina, } 13.650 \mathrm{mg} \text {; piridoxina, } 700 \\
\text { mg; riboflavina, 2.100 mg; selênio, } 136,5 \mathrm{mg} \text {; tiamina, } 700 \mathrm{mg} \text {; vit. A, } \\
2.800 \text { Ul; vit. B12, } 11.550 \mathrm{mcg} \text {; vit. D3, } 1.050 \mathrm{UI} \text {; vit. E, } 10.500 \mathrm{mg} \text {; vit. } \\
\text { K3, } 2.800 \mathrm{mg} ; \text { zinco, } 77.999 \mathrm{mg} \text {; cobre, } 15.750 \mathrm{mg} \text {; manganês, } 41.850 \\
\text { mg; cálcio, } 98.800 \mathrm{mg} \text {; cobalto, } 135 \mathrm{mg} \text {; iodo, } 1.470 \mathrm{mg} \text {; ferro, } 26.250 \mathrm{mg} \\
\text { (valores por kg de produto). }\end{array}$ \\
\hline $\begin{array}{l}\text { Mistura vitamínica e mineral } \\
\text { para a fase crescimento }\end{array}$ & $\begin{array}{l}\text { Vit. A } 8.333 \text { Ul; ácido nicotínico, } 25.000 \text { mg; selênio, } 208,33 \text { mg; vit. D3, } \\
1.875 \text { Ul; ác. Pantotênico, } 12.500,0 \text { mg; iodo, } 406,25 \mathrm{mg} \text {; vit. E, } 33.333 \\
\text { Ul; biotina, } 104,17 \text { mg; veículo q.s.p., } 1.000,0 \text { g; vit. K3, } 1.250,0 \mathrm{mg} \text {; ácido } \\
\text { fólico, } 500,0 \mathrm{mg} \text {; vit. B1, } 1.667,0 \mathrm{mg} \text {; ferro, } 54.167,0 \mathrm{mg} \text {; vit. B2, } 4.167,0 \\
\text { mg; cobre, 8.125,0 mg; vit. B6, } 2.500,0 \mathrm{mg} \text {; zinco, } 65.000,0 \mathrm{mg} \text {; vit. B12, } \\
20,83 \mathrm{mg} \text {; manganês, } 32.500,0 \mathrm{mg} \text { (valores por kg de produto). }\end{array}$ \\
\hline Dieta pré-inicial peletizada & $\begin{array}{l}\text { Milho, 40,9\%; farelo de soja, 33,5 \%; soro de leite em pó, } 10,0 \% \text {; lacto- } \\
\text { se, 7,0 \%; calcário, 0,52 \%; fosfato bicálcico, 2,09 \%; óleo de soja, 4,04 } \\
\% \text {; L-Lisina, 0,30 \%; DL-Metionina, 0,15\%; L-Treonina, 0,03 \%; NaCl, } \\
\text { 015\%; promotor de crescimento, 0,50\%; cloreto de colina (60\%), 0,20\%; } \\
\text { mistura vitamínica e mineral, 0,60\%; antioxidante, 0,01\%. }\end{array}$ \\
\hline Dieta crescimento peletizada & $\begin{array}{l}\text { Milho }(8,2 \% \text { PB), } 52,71 \% \text {; farelo de soja, } 22,03 \% \text {; glúten de milho, } 5,61 \\
\% \text {; fosfato bicálcico, } 1,63 \% \text {; calcário, } 1,09 \% \text {; mistura mineral, } 0,10 \% \text {, } \\
\text { mistura vitamínica, } 0,10 \% \text {; cloreto de sódio, } 0,34 \% \text {; BHT, 0,01 \%; óleo de } \\
\text { soja, } 1,83 \% \text {; amido, } 18,15 \% \text {; material inerte } 0,83 \% \text {. }\end{array}$ \\
\hline
\end{tabular}

A acidez ou alcalinidade tituláveis (AT) foram calculadas segundo a metodologia utilizada por GIGER-REVERDIN et al. (2002), definida como a quantidade de ácido ou base requerida para alterar o pH entre o pHi e 5,0, com os resultados expressos em $\mathrm{mEq} / 100 \mathrm{~g}$ de matéria seca (MS) do alimento. A capacidade tamponante (CT) é então calculada dividindo os valores de AT pelo intervalo de $\mathrm{pH}$ considerado na determinação. Não se observou diferença entre os ácidos usados na titulação para AT e CT ( $p>0,99)$, sendo assim os valores obtidos com cada ácido foram considerados como replicatas para cada amostra. A MS dos alimentos foi medida em estufa de ar forçado à temperatura de $105^{\circ} \mathrm{C}$ até peso constante. Os resultados das medidas de $\mathrm{pHi}$,
AT e CT foram submetidos à análise de variância considerando as classes de ingredientes. As médias foram comparadas pelo teste de Duncan com nível de significância de $5 \%$, e foi medida a correlação linear entre o $\mathrm{pHi}$ e a CT.

\section{RESULTADOS E DISCUSSÃO}

Na TABELA 1 estão apresentados os resultados obtidos por material estudado.

Os valores de $\mathrm{pHi}$, AT e CT variaram de 3,35 a $8,91,0,49$ a 120,30 (mEq/100 g MS) e 0,12 a 88,30 (AT/intervalo de $\mathrm{pH}$ ), respectivamente.

As amostras de fosfato bicálcico, Premix2 e treonina destacaram-se por apresentar valores de $\mathrm{pHi}$ menores que 5,0 , de $3,35,4,12$ e 4,50 respectiva- 
mente. Já a o $\mathrm{NaCl}$ mostrou pHi elevado, com um valor médio de 8,91. Esses valores foram diferentes dos obtidos por LAWLOR et al. (2005), que registraram valores de $\mathrm{pHi}$ de 7,5 para $\mathrm{NaCl}, 6,5$ para a treonina e 6,5 para a lisina. Essas diferenças podem ser atribuídas a diferenças de composição nos lotes de ingredientes e às condições de análise (temperatura, água, etc.). $\mathrm{O}$ pHi obtido para as amostras de milho, farelo de soja e soro de leite em pó foram similares aos registrados por GIGER-REVERDIN et al. (2002) e VAN OOIJEN (2002). LAWLOR et al. (2005) obtiveram valor de $\mathrm{pHi} 0,8$ pontos mais alto para uma amostra de milho, e valores de 5,2 para duas pré-misturas vitamínicas e minerais para fases pré-inicial e crescimento. Para a medida AT (mEq/100 g MS), o NaCl, a quirera de arroz e os aminoácidos apresentaram valores menores que 2 , enquanto farinha de carne, o plasma e o Premix2 apresentaram valores maiores que 30 , chegando a 120 para o fosfato de cálcico. A ordem de classificação dos materiais quanto à AT está coerente com os resultados de LAWLOR et al. (2005), que mediram a AT até o $\mathrm{pH} 4$ e 3.

TABELA 1 - MATERIAL, NÚMERO DE AMOSTRAS DE CADA MATERIAL (N) E VALORES MÉDIOS E DESVIO PADRÃO DE PH INICIAL (PHI), ACIDEZ OU ALCALINIDADE TITULÁVEIS (AT) E CAPACIDADE TAMPONANTE (CT).

\begin{tabular}{|c|c|c|c|c|}
\hline Material & $\mathrm{N}$ & $\mathrm{pHi}$ & $A T^{1}$ & $\mathrm{CT}^{2}$ \\
\hline Lisina & 1 & $5,70 \pm 0,04$ & $1,00 \pm 0,08$ & $1,43 \pm 0,15$ \\
\hline Metionina & 2 & $5,93 \pm 0,24$ & $1,35 \pm 0,75$ & $1,42 \pm 0,55$ \\
\hline Treonina & 1 & $4,50 \pm 0,01$ & $1,53 \pm 0,01$ & $2,84 \pm 0,01$ \\
\hline Farinha de carne & 2 & $7,14 \pm 0,26$ & $38,18 \pm 2,90$ & $17,31 \pm 1,67$ \\
\hline Farinha de penas & 2 & $6,12 \pm 0,15$ & $11,61 \pm 2,38$ & $10,86 \pm 3,36$ \\
\hline Farinha de vísceras & 3 & $6,38 \pm 0,06$ & $29,38 \pm 3,99$ & $21,68 \pm 3,21$ \\
\hline Plasma & 1 & $6,83 \pm 0,22$ & $44,57 \pm 2,71$ & $24,48 \pm 4,37$ \\
\hline Soro de leite em pó & 1 & $6,40 \pm 0,01$ & $12,70 \pm 0,98$ & $9,13 \pm 0,68$ \\
\hline Far. de arroz integral & 1 & $6,63 \pm 0,06$ & $29,51 \pm 0,88$ & $18,18 \pm 0,56$ \\
\hline Far. de arroz deseng. & 1 & $6,47 \pm 0,06$ & $25,85 \pm 2,90$ & $17,80 \pm 1,67$ \\
\hline Milho & 3 & $5,96 \pm 0,10$ & $3,36 \pm 0,60$ & $3,44 \pm 0,39$ \\
\hline Quirera de arroz & 1 & $6,48 \pm 0,04$ & $1,92 \pm 0,65$ & $1,30 \pm 0,47$ \\
\hline Dieta cresc. pelet. & 2 & $5,98 \pm 020$ & $11,50 \pm 1,20$ & $12,21 \pm 2,46$ \\
\hline Dieta pré-inicial pelet. & 1 & $6,27 \pm 0,03$ & $11,04 \pm 0,94$ & $8,78 \pm 0,58$ \\
\hline Resíduo de biscoito & 1 & $6,40 \pm 0,02$ & $9,86 \pm 0,51$ & $7,09 \pm 0,24$ \\
\hline Fosfato bicálcico & 1 & $3,35 \pm 0,002$ & $120,30 \pm 0,01$ & $72,95 \pm 0,02$ \\
\hline Mist. vit. e min. cresc. & 1 & $4,12 \pm 0,02$ & $77,44 \pm 0,01$ & $88,30 \pm 0,01$ \\
\hline Mist. vit. e min. pré-inic. & 1 & $5,60 \pm 0,05$ & $11,88 \pm 1,73$ & $19,66 \pm 1,38$ \\
\hline $\mathrm{NaCl}$ & 1 & $8,91 \pm 0,15$ & $0,49 \pm 0,04$ & $0,12 \pm 0,01$ \\
\hline Farelo de Soja & 3 & $6,40 \pm 0,04$ & $29,21 \pm 0,95$ & $21,06 \pm 0,63$ \\
\hline Soja integral & 1 & $6,32 \pm 0,09$ & $17,27 \pm 0,79$ & $12,94 \pm 0,11$ \\
\hline Soja integral tostada & 1 & $6,45 \pm 0,04$ & $17,50 \pm 1,59$ & $12,10 \pm 0,75$ \\
\hline
\end{tabular}

${ }^{1} \mathrm{mEq} / 100 \mathrm{~g} \mathrm{MS} ;{ }^{2} \mathrm{AT} /$ intervalo $\mathrm{pH}$ 
matérias-primas e dietas utilizadas para leitões

Os valores mais altos de CT (AT/intervalo de $\mathrm{pH}$ ) foram obtidos com o fosfato bicálcico e o Premix2, maiores que 70 , enquanto os mais baixos, menores que 4, foram obtidos com quirera de arroz, o milho e os aminoácidos. Estes valores estão dentro da expectativa a partir dos resultados publicados por JASAITIS et al.(1987) e LAWLOR et al. (2005).
$\mathrm{Na}$ TABELA 2 são apresentados os resultados de acordo com a classificação dos materiais estudados. Dentre os aminoácidos e os suplementos minerais houve amostras com valores de $\mathrm{pHi}$ superiores e inferiores a 5,0 , e com base nisso essas classes de materiais foram subdivididas em "ácidos" e "neutros".

TABELA 2 - VALORES MÉDIOS E DESVIO PADRÃO DE PH INICIAL (PHI), ACIDEZ OU ALCALINIDADE TITULÁVEIS (AT) E CAPACIDADE TAMPONANTE (CT) PARAAS CLASSES DE INGREDIENTES E DIETAS ANALISADOS 1 .

\begin{tabular}{cccc}
\hline CLASSES & $\mathrm{pHi}$ & $\mathrm{AT}^{2}$ & $\mathrm{CT}^{3}$ \\
\hline Soja e subprodutos & $6,40 \pm 0,60 \mathrm{cb}$ & $24,48 \pm 1,11 \mathrm{~b}$ & $17,64 \pm 0,50 \mathrm{~b}$ \\
Cereais e subprodutos & $6,24 \pm 0,06 \mathrm{cb}$ & $11,23 \pm 1,09 \mathrm{c}$ & $7,93 \pm 0,66 \mathrm{~cd}$ \\
Ingr. de origem animal & $6,54 \pm 0,14 \mathrm{~b}$ & $27,22 \pm 2,59 \mathrm{~b}$ & $17,22 \pm 2,66 \mathrm{~b}$ \\
Aminoácidos "neutros" & $5,85 \pm 0,14 \mathrm{c}$ & $1,23 \pm 0,41 \mathrm{c}$ & $1,42 \pm 0,35 \mathrm{e}$ \\
Aminoácidos "ácidos" & $4,50 \pm 0,05 \mathrm{~d}$ & $1,53 \pm 0,20 \mathrm{c}$ & $2,84 \pm 0,16 \mathrm{de}$ \\
Supl. minerais "neutros" & $7,26 \pm 0,10 \mathrm{a}$ & $6,19 \pm 0,88 \mathrm{c}$ & $9,89 \pm 0,69 \mathrm{c}$ \\
Supl. minerais "ácidos" & $3,73 \pm 0,05 \mathrm{e}$ & $98,87 \pm 14,45 \mathrm{a}$ & $83,63 \pm 6,36 \mathrm{a}$ \\
Dietas & $6,16 \pm 0,08 \mathrm{cb}$ & $10,97 \pm 0,88 \mathrm{c}$ & $10,07 \pm 1,10 \mathrm{c}$ \\
\hline
\end{tabular}

${ }^{1}$ Médias seguidas de letras distintas na coluna diferem pelo teste de Duncan $(P<0.05) ;{ }^{2} \mathrm{mEq} / 100 \mathrm{~g}$ matéria seca; ${ }^{3} \mathrm{AT} /$ intervalo de $\mathrm{pH}$.

Os minerais "ácidos" e "neutros" apresentaram respectivamente o menor e maior valor médio de $\mathrm{pHi}$ $(P<0,05)$. As dietas, os cereais, a soja e seus subprodutos apresentaram valores similares de $\mathrm{pHi}$, que não foram significativamente diferentes dos obtidos para os aminoácidos "neutros" e os ingredientes de origem animal $(P>0,05)$. Os aminoácidos "ácidos" apresentaram valores de $\mathrm{pHi}$ intermediários entre os dois grupos $(P<0,05)$. Com a medida AT as classes foram separadas em três grupos, com menores valores para os suplementos minerais "ácidos", seguidos pelos produtos da soja e os ingredientes de origem animal, com valores intermediários, sendo as demais classes agrupadas com os mais baixos valores $(P<0,05)$. A medida de CT mostrou maior sensibilidade que a AT, resultando em diferença significativa entre os aminoácidos "neutros" e as classes suplementos minerais "neutros", cereais e dietas. BOLDUAN et al. (1988) observaram que o aumento da suplementação mineral na dieta de 0 a $4 \%$ triplicou o valor de AT da mesma, o que está de acordo com os elevados valores de CT em minerais registrados por LAWLOR et al. (2005). PROHASZKA e BARON (1980) observaram que os valores de CT aumentaram para ingredientes com maiores níveis de proteína. No presente trabalho, excetuando-se os suplementos minerais e os aminoácidos, as classes com maiores AT e CT foram os suplementos protéi- cos, que poderiam ser agrupados dentro da mesma classe, com exceção, talvez, de ingredientes com alto nível de cálcio, como algumas farinhas de carne (TABELA 1).

A correlação entre o pHi e a CT foi negativa incluindo-se os aminoácidos e os minerais, mas foi positiva com a retirada dessas classes de amostra (FIGURA 1). A inconsistência da correlação demonstra que o pHi não é estimativa confiável da CT.

FIGURA 1 - CORRELAÇÃO ENTRE O pH INICIAL E A CAPACIDADE TAMPONANTE.

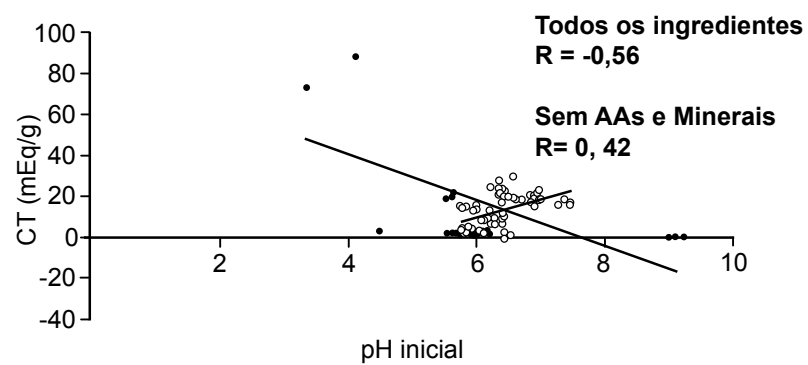

Archives of Veterinary Science, v 12, n.3. p. 71-76, 2007 


\section{CONCLUSÃO}

A medida de capacidade tamponante descreve melhor as diferenças entre alimentos e dietas do que o $\mathrm{pH}$, em solução, e a acidez ou alcalinidade tituláveis. Sugere-se a formação de subclasses para alguns materiais, como minerais e aminoácidos, que apresentaram valores extremos para as todas variáveis mensuradas. $\mathrm{O} \mathrm{pH}$ inicial não demonstrou uma correlação consistente com a capacidade tamponante, não sendo, portanto, uma boa estimativa para tal característica.

\section{REFERÊNCIAS}

BACCAN, N.; DEANDRADE, J.C.; GODINHO, O.E.S., BARONE, J.S. Química Analítica Quantitativa Elementar. Campinas: Edgard Blucher, 1991. 259 p.

BOLDUAN, G.; JUNG, H.; SCHNABEL, E.; SCHNEIDER, R. Recent advances in the nutrition of weaner pigs. Pig News and Information, v. 9, p. 381-385, 1988.

GABERT, V. M.; SAUER, W. C.; SCHMITZ, M.; AHRENS, F.; MOSENTHIN, R. The effect of formic acid and buffering capacity on the ileal digestibilities of amino acids and bacterial populations and metabolites in the small intestine of weanling pigs fed semipurified fish meal diets. Journal of Animal Science, v. 75, p. 615-623, 1995.

GIGER-REVERDIN,.S.; PONTER,D.C.; SAUVANT,D.; MARTIN, O.; PRADO, N.I.; MÜLLER,R. Intrinsic buffering capacity of feedstuffs. Animal Feed Science and Technology, v.96, p.83-102, 2002.

JASAITIS, D.K.; WOHLT, J.E.; EVANS, J.L. Influence of feed-ion content on buffering capacity of ruminant feedstuffs in vitro. Journal of Dairy Science, v.70, p. 1391-1403, 1987.

LAWLOR, P.G.; LYNCH, P.B.; CAFFREY, P.J.; O' REILLY,J.J.; O'CONNELL,M.K. Measurements of the acid-binding capacity of ingredients used in pig diets. Irish Veterinary Journal, v.58, p. 447-452, 2005.

McBURNEY,M.I.; VAN SOEST,P.J.; CHASE,L.E.; Cation exchange capacity and buffering capacity of neutral-detergent fibers. Journal of Sciencie and Food Agricultural, v. 34, p. 910-916, 1983.
OLIVEIRA Jr, J.M.; WARPECHOWSKI, M.B. Avaliação da aditividade de medida linearizada da capacidade tamponante da fibra em alimentos. In: I SIMPÓSIO SUL BRASILEIRO DE OVINOS E CAPRINOS. 2007, Curitiba, Anais... Curitiba: Universidade Federal do Paraná, 2007.

PARTANEN, K.; SILJANDER-RASI, H.; ALAVIUHKOLA, T.; SUOMI, K.; FOSSI, M. Performance of growing-finishing pigs fed médium or high-fibre diets supplemented with avilamycin formic acido or formic acid-sorbate blend. Livestock Production Science, v.73, p. 139-152, 2002.

PENZ Jr,A.M. Hipótesis que jutifican el uso de acidos orgânicos em lãs dietas para aves y cerdos. Avicultura Profesional, v.9, n.1,p.46-51, 1991.

PROHASZKA, L.; BARON, F. The predisposing role of high dietary protein supplies in enteropathogenic $E$. coli infections of weaned pigs. Zentralblatt für Veterinärmedizin, v. 27, p. 222-232, 1980.

RISLEY, C. R.; KORNEGAY, E. T.; LINDEMANN, M. D.; WOOD, C. M.; EIGEL, W. N. Effect of feeding organic acids on selected intestinal content measurements at varying times postweaning in pigs. Journal of Animal Science, p. 196-206, 1992.

ROTH, F.X. Ácidos orgánicos en nutrición porcina: eficacia y modo de acción. In: CURSO DE ESPECIALIZACIÓN, 11, 2000 [S.I.]. Proceedings... [S.I.]: Fundación Española para el Desarrollo de la Nutrición Animal - FEDNA, 2000,p.169-181.

VAN OOIJEN, C. Quais os efeitos do Calprona $P$ adicionado a ração? Boletim técnico. Editora Verdugt. Porto Alegre. 2002.

WARPECHOWSKI, M. B.; CIOCCA, M. L. S. Propriedades físico-químicas da fibra em detergente neutro de alimentos isolados e misturados. Archives of Veterinary Science, v.11, n.1, p.23-26. 2006.

WARPECHOWSKI, M.B.; SILVA, D.A. Modificação de técnica de determinação da capacidade de troca catiônica da fibra em detergente neutro de alimentos. Archives of Veterinary Science, v. 11, n. 2, p. 30-33, 2006.

Recebido para publicação: $\quad$ 01/06/2007 Aprovado: $18 / 12 / 2007$ 